\title{
Propriospinal neurons old but not forgotten
}

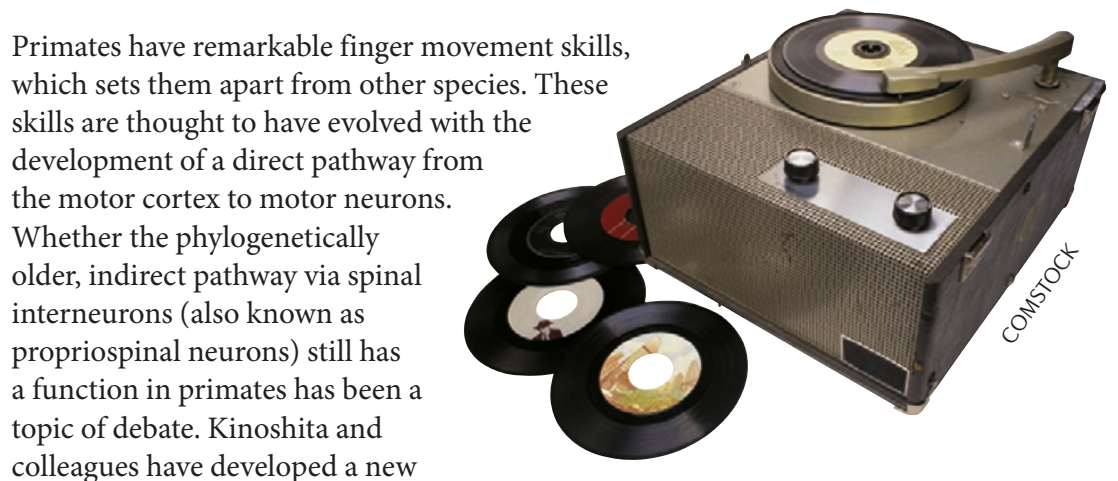
colleagues have developed a new technique to temporarily and selectively inactivate spinal interneurons, and show that these neurons are required for reach and grasp movements.

The authors set out to assess the function of the indirect pathway by inactivating the relevant spinal interneurons in macaque monkeys. For this purpose they created two viral vectors. The first was a retrograde lentiviral vector carrying a tetracycline-responsive element and, downstream from this, genes encoding green fluorescent protein and enhanced tetanus neurotoxin light chain (eTeNT). The second viral vector was an adeno-associated virus serotype 2 vector carrying the Tet-On sequence under the control of the cytomegalovirus promoter. Thus, in a double-infected cell, transcription of Tet-On would - in the presence of the tetracycline analogue doxycycline - induce the expression of eTeNT, and this would prevent neurotransmission in that cell. This approach allows for the inactivation of neurons in a manner that is both specific (only in cells that are transfected with both viral vectors) and temporally regulated (only after doxycycline administration).

The authors injected the first viral vector into the spinal cord region that contains the hand-arm motor neurons that are targeted by the spinal interneurons. After 7-10 days, they injected the second vector into the region of the spinal cord where the cell bodies of the spinal interneurons are located.

Administration of doxycycline 1-2 months after the double infection resulted in impaired reach and grasp movements within $2-5$ days. This impairment was resolved within 2-3 days, suggesting that the direct pathway may have compensated for the loss of function of the propriospinal neurons. When the authors terminated doxycycline treatment and restarted it 3-4 weeks later, the monkeys again showed reach and grasp impairments. This indicates that the functional compensation (presumably by the direct pathway) was only temporary and that the indirect pathway regained function after doxycycline treatment was stopped.

In addition to the finding that the phylogenetically old, indirect pathway is still functional in monkeys, a major contribution of this study is the development of a technique that makes it possible to temporarily inactivate a select population of neurons - and thus to study neural circuits - without the need for transgenic animals. Creating transgenic animals for circuit dissection is only possible if neuronal subtype-specific promoters for the circuit of interest are known and is difficult in larger animals. The new technique therefore provides an alternative, powerful tool for the study of neural circuits in primates and other species.

Leonie Welberg 